\title{
Use of a Smartphone to Improve Hospital Discharge Plan Service
}

\author{
Liarn Rurng Wen and Ching Miin Duh
}

\begin{abstract}
Discharge plan service, which affects patients' recovery ratio and hospitals' bed turnover rate is very significant in hospital revenues in recent years. However, the effectiveness of discharge plan service has received little attention. Case managers and clinic specialists in hospitals require the latest clinical history and reports of patients anytime and anywhere in order to promote healthcare quality. Therefore the shift from health information system (HIS) to mobile medical service system is an unavoidable trend. This article reports the implementation of an intelligent Hospital Discharge Plan Services system, which integrates a smartphone through the hospital wireless network to store/access clinical data without time and space limitation. Based on master-slave architecture, Intelligent Hospital Discharge Plan Services (IHDPS) enables health professionals to access back-end patients' clinical data from the smartphone via an internet connection. IHDPS, through the provision of sufficient patients' physical information from a clinical database, helps case managers can employ decision tree algorithm to evaluate patients' comprehensive conditions for adequate discharge arrangement. The system also reduces the workload of healthcare professionals, promotes healthcare quality and reduces the frequency of patients' in and out of hospital, hence increasing bed turnover rate.
\end{abstract}

Index Terms-Hospital discharge plan, organizational performance, healthcare information system, smartphone.

\section{INTRODUCTION}

In Taiwan, stroke is one of the main diseases that cause disability in long-term care. Because the situation is that active neural rehabilitation and complete medical care of stroke patients are needed to prevent stroke recurrence. During this time in hospital, healthcare professionals and case managers have to examine patients' physical condition and fill in daily inspection reports in a traditional clinical record paper [1]. The disadvantages of the situation could be due to the inefficient way of records input, followed by difficulty of records maintenance and finally, the review treatment process for discharge plan services by healthcare professionals is a tedious job because of insufficient and ineffective structured data to used [2]. Many health care information systems were implemented to provide features that can overcome problems such as mobile nursing cart [3]. Therefore, developing an information system which can solve the above problems and

Manuscript received April 1, 2014; revised December 3, 2014.

Liarn Rurng Wen is with the Department of Management Information Systems, Meiho University, Ping Tung, Taiwan (e-mail: x00003029@meiho.edu.tw).

Ching Miin Duh is with the Department of Information Management, National Taipei University of Nursing and Health Sciences, Taipei, Taiwan (e-mail: chingmiin@ntunhs.edu.tw). can provide automated information becomes impending challenges for health care informatics and health care professionals.

With technology advancement, smart phones have many characteristics such as small size, lightweight, easy to carry, and cheaper than a laptop. Moreover, they are equipped with wireless LAN Wi-Fi high-speed transmission and high-capacity memory among others, making them powerful tools in medical informatics. Many research reported [4] that there are many medical informatics systems, but few integrate with features of smart phone and focus on discharge service plan. The purpose of this study is to integrate smart phone and web technology to develop an IHDPS system. With IHDPS, healthcare professionals can input patients' physical conditions without carrying a volume of the clinical record. IHDPS also provides data access feature at any place as long as there is an internet connection. In addition, it provides clinical data entry and query function, which help case managers to determine patients' physical condition for hospital discharge plan efficiently. IHDPS can effectively reduce healthcare professional workload, and improve the quality of medical care, leading to an increase of hospital revenue.

\section{LITERATURE REVIEW}

\section{A. The Situation and Difficulty of Stroke Patients Care}

Stroke is caused by loss of brain function due to sudden obstruction in the blood flow. Stroke patients often suffer from infections, pressure ulcers, cramps, vomiting, seizures (convulsions), and other symptoms. Care and therapy through physical therapy and occupation therapy methods are, therefore, needed to help patients recover to normal daily function in a short period [3]. Nurses and healthcare professional play a pivotal role in the discharge planning process of stroke patients [5]. According to research conducted in Taiwan, about $80 \%$ of nurses agreed that "excessive workload" was the biggest problem in their workplace. $90 \%$ of nurses believe that if they have the opportunity to spend more time to take care patients, then the healthcare quality will have a significant impact. Based on Taiwan Ministry of Economic Affairs report in 2008 [6], the new medical problem is from aging society and low birth rate, and the trend of medical care division causes the shortage of labor resources. Therefore, through an organized systematic decision-making actions, case management becomes a professional service design. Case management model not only ensures the safety of patients after discharge but also enhances the quality of the life of patients and their families. From 
hospital management aspect, it can help reduce patient readmission rates, reduce the cost of health care expenditure, and raise the revenue of the hospital.

\section{B. Discharge Plan Services Process for Stroke Patients}

Once a patient is admitted into the hospital, the healthcare professional will assess and evaluate (first evaluation) the patient's physical condition within the first 24 hours while the case manager will perform the second assessment within the next 72 hours. The healthcare professional will usually regularly assess the condition of stroke patients. If the patient is in a stable condition and reaches discharge standards, then the healthcare professional will assess which model is the best arrangement for the patient. If there is no need for intubation, then the patient can be discharged for home care. However, if the patient is in abnormal physical conditions, then there is a need to do a different arrangement, such as a nursing home [4], [7], [8].

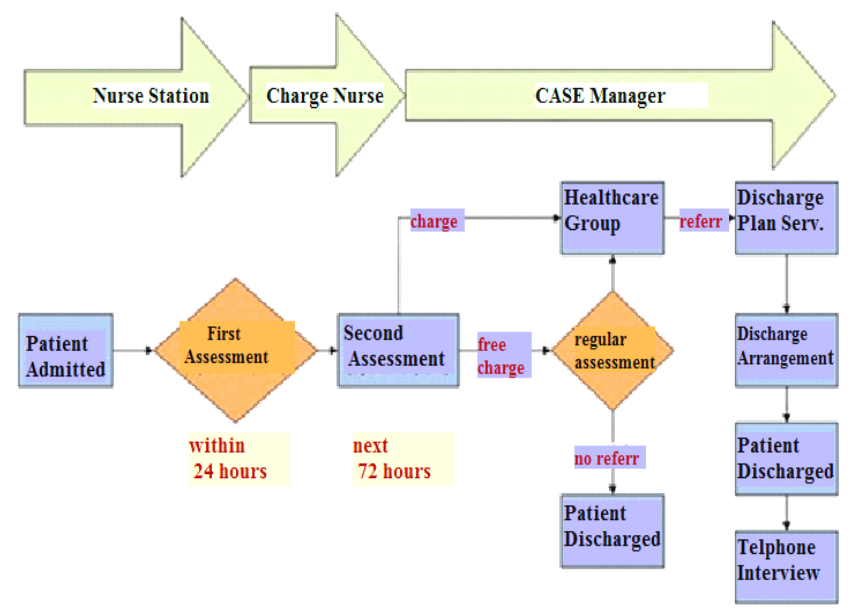

Fig. 1. Discharge plan service process for stroke patient.

\section{The Role of Information Technology in Discharge Plan Service}

The American Hospital Association defines discharge planning as a centralized coordination and multidisciplinary process. Through healthcare professionals, families work together to ensure that patients get continuity of care after discharge; plan must reflect the social, emotional, medical and psychological needs from the patients and their families [9]. In order to enhance the efficiency of discharge plan service, information technology becomes the key element of the task. With advance smart phone devices and user-friendly case management information system, healthcare professionals are able to track and maintain patient's clinical record over time and space barrier. Buss [10] pointed out that the objective of the information technology strategy is to formulate a long-term plan of an information system. Earl [11], [12] reported that the architecture of an information technology is the blueprint of the use of that information technology. He indicated that information technology architecture has four elements: 1) Computing: hardware and operating system, 2) Communications: network, hardware and software. 3) Data: valuable information of an enterprise, and the hardware, software facilities that can process those data. 4) Applications: enterprise application systems, including systems development methods.

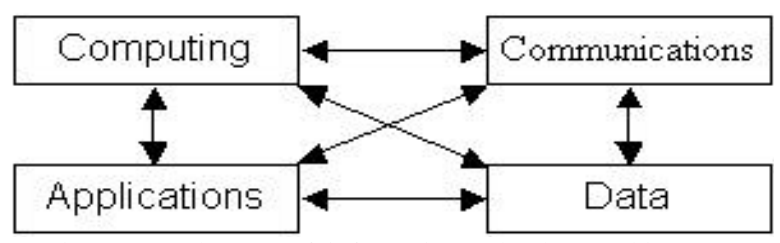

Fig. 2. Four elements of information technology architecture.

\section{SYSTEM ARCHITECTURE OF IHDPS}

There are five system modules in IHDPS includes 1) Vital signal and health data management module, 2) Post-hospital assessment and transfer module and 3) Post-hospital resources integration module. Fig. 3 illustrates the data flow and relationships among three modules.

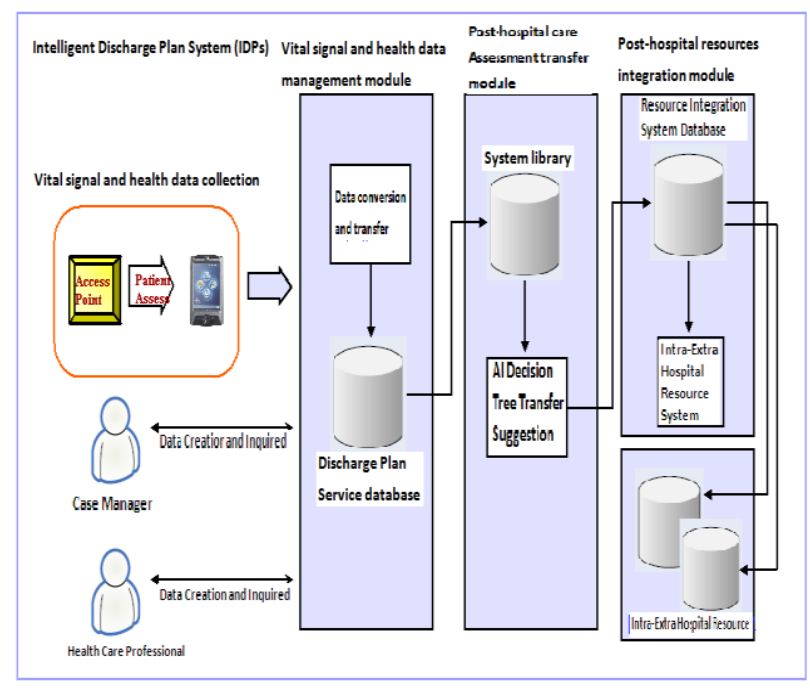

Fig. 3. System architecture of IHDPS.

The main tasks of each module are as following:

\section{1) Vital signal and health data management module}

The main task of this module is to use smart phone to develop a dynamic effective system which can collect in or out patients' vital signal and health data. Hospital case manager, home care and community nursing professions can perform better care service and post-hospital tracking based on collected data. The data will be saved onto hospital discharge plan service system for statistic analyzing. This system provides monitoring feature for patients, it can forward alert message to nursing professional when patients' vital signal or health data are abnormal.

\section{2) Post-hospital health care assessment and transfer} suggestion system

This system generates transfer suggestions for patient discharge plan. The system uses decision tree technique of artificial intelligence to assess patient's need according to patient's vital signal, health record and individual's social support. The final assessment result provides useful information for transfer arrangement.

\section{3) Post-hospital resource integration module}

The main purpose of this module is to integrate resources in the hospital and off the hospital according to the needs of patients in different stages. Case manager may allocate appropriate resources and best-fit healthcare services through the in-off hospital database network system in a short instance. 
Moreover, the system provides the feature of transfer the patients' medical record to the indicated post-hospital healthcare unit database system, thus it enhances the efficient of the preparation process for new coming patients and as well as can reduce the patients' coping time to the new environment.

\section{SYSTEM IMPLEMENTATION}

\section{A. Network Architecture of IHDPS}

The IHDPS system is implemented in a medical center in Taipei, Taiwan, the medical center is in the level 5 hospital accreditation, there are 3 wards and total 100 patients in internal neural department, the average annual cases amount is 1000 , but only 3 case managers in charge of discharge plan services. Before the system employed, the case managers have to record patients' health condition, physiological needs, medical supporting system needs, status quo of economic and further information in healthcare needed onto paper chart for evaluation and resources allocation. Once completed the paper case-management chart, the data will be keyed into a simple excel case management system for data storage and usage or later healthcare treatments. In order to improve the bedside healthcare needs information collecting flow, the data collection feature of IHDPS was designed and implemented on a smartphone, whereas the statistical data analyzing, discharge plan and data storage features were implemented in a dedicated server. Fig. 4 illustrates the network architecture of an intelligent hospital discharge plan system.

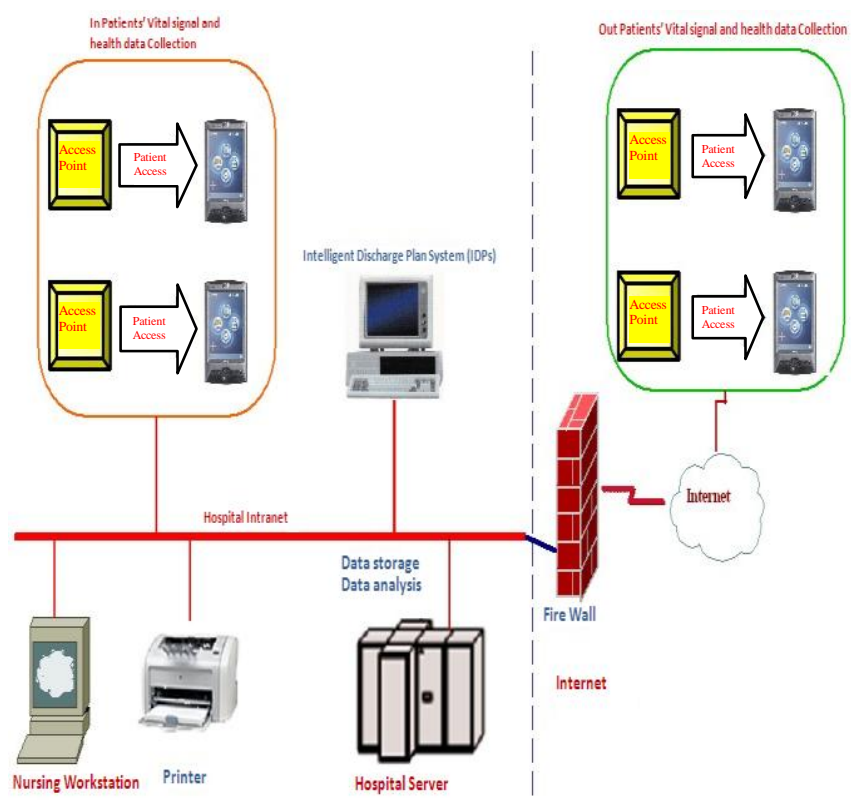

Fig. 4. Network architecture of intelligent hospital discharge plan system.

Fig. 4 shows while case managers apply smartphone to collect the health condition, physiological needs and healthcare equipment needs of patients, and then transfer the data via hospital Wi-Fi system (asynchronous) or to perform a synchronous data collection via hospital network system into IHDPS. Moreover, case managers can apply discharge transfer decision support system to generate transfer suggestions according to patients' baseline data. The results will be saved in the hospital main server and integrate onto patients' medical record for further reference. The health baseline record will be transferred to post-hospital unit for healthcare professionals to perform post-hospital healthcare services.

\section{B. System Implementation Environment and Tools}

IHDPS is implemented on HTC HD2 smartphones, mainly because they are lightweight, convenient, and have many features, including internet, Wi-Fi wireless and scalability.

TABLE I: IHDPS IMPLEMENTATION ENVIRONMENT AND TOOLS

\begin{tabular}{|c|c|c|c|c|c|}
\hline & $\begin{array}{c}\text { System } \\
\text { develop tools }\end{array}$ & Language & Database & Platform & $\begin{array}{c}\text { Graphic } \\
\text { tools }\end{array}$ \\
\hline $\begin{array}{l}\text { Smart } \\
\text { Phone } \\
\text { Apps }\end{array}$ & $\begin{array}{l}\text { Microsoft } \\
\text { Visual Studio } \\
\text { 2008Windows } \\
\text { Mobile } 6.0 \\
\text { Pocket } \\
\text { PC SDK }\end{array}$ & $\mathrm{C \#}$ & \multirow{2}{*}{$\begin{array}{l}\text { Micro-so } \\
\text { ft SQL } \\
\text { Server } \\
2005\end{array}$} & \multirow{2}{*}{$\begin{array}{l}\text { Internet } \\
\text { Informati } \\
\text { on } \\
\text { Services } \\
7.0\end{array}$} & \multirow[t]{2}{*}{$\begin{array}{l}\text { Photo } \\
\text { Impact } \\
12\end{array}$} \\
\hline $\begin{array}{l}\text { Web } \\
\text { page }\end{array}$ & $\begin{array}{l}\text { Microsoft } \\
\text { Visual Web } \\
\text { Developer } \\
\text { 2005 Express }\end{array}$ & $\begin{array}{l}\text { ASP.NE- } \\
\mathrm{T} \\
3.5\end{array}$ & & & \\
\hline
\end{tabular}

\section{Cloud Client - Server IHDPS System Structure}

Healthcare professionals can log into computers and smart mobile devices either in the hospital or outside the hospital through network connection to the web server. After that, they can operate IHDPS to inquire patients' record or perform physical condition assessment and then store patients' clinical records into the database server. Fig. 5 presents the database server structure of IHDPS.

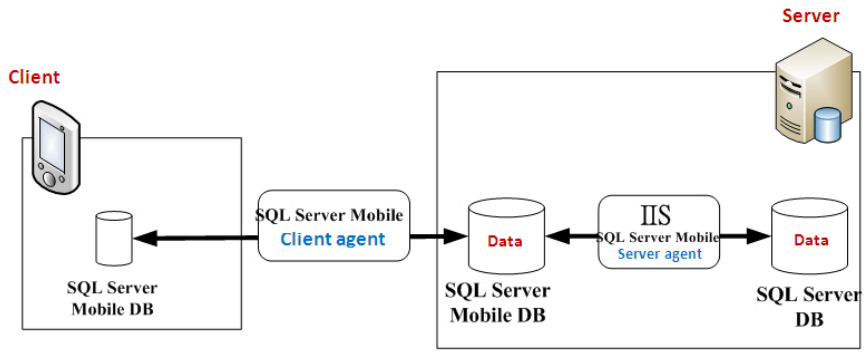

Fig. 5. Database server structure of IHDPS.

The data storage of IHDPS system is Microsoft SQL Server 2005 in which data can be retrieved and accessed via Wi-Fi wireless networks. The architecture is divided into the server side and the client side. The server side comprises of two types of database, the SQL server database and the SQL server mobile database. These two types of database use IIS SQL server mobile (server agent) to process synchronous data updating. With client-side (Smart Phone side) on the other hand, the system applies a simple SQL server mobile database. When IHDPS disconnected from the server, the data would be stored onto smart phone database.

\section{RESULTS AND CONCLUSIONS}

The main purpose of this study is to report the implementation of an intelligent hospital discharge plan services (IHDPS). IHDPS system integrates smartphone, internet and database technology to enable health professionals to access back-end patients' clinical records via smart phone as long as there is an internet connection. It also 
allows healthcare professionals to input and inquire patients' physical condition for discharge assessment, hence overcoming time and space barrier. Patients physical condition assessment function, enables case managers to process discharge plan service efficiently. From the hospital management aspect, it can promote healthcare quality, reduce patient readmission rates, raises hospital bed turnover rate, reduce the cost of healthcare expenditure and raise the revenue of the hospital.

\section{REFERENCES}

[1] Z. R. Jun, "Development and evaluation of the comprehensive care of ischemic stroke clinical pathway," M.S. Thesis, Dept. Healthcare Management, China Medical College, Taichung, Taiwan, 2003.

[2] T. F. Wu and Q. Xie, Information Management - Theory and Practice, 3rd ed., Taipei: Taiwan, 1998, pp. 134-136.

[3] H. Xu, "Personal digital assistants (PDAs) based wearable fall real-time monitoring system," M.S. Thesis, Dept. Automatic Control Engineering, Feng Chia University, Taichung, Taiwan, 2002.

[4] S. Lin, "The construction of the discharge planning follow-up care information systems, case-based reasoning," M.S. Thesis, Dept. Industrial Engineering and Management Institute, National Yunlin University of Science and Technology, Yunlin, Taiwan, 1992.

[5] P. Kerr, "Stroke rehabilitation and discharge planning," Nurs Stand., vol. 27, no. 1, pp. 35-39, Sep. 2012.

[6] Ministry of Economic Affairs and Investment Bureau. The health care industry and investment opportunities. [Online]. Available: http://www.taiwantrade.com.tw/MAIN/resources/MAIN/TC/ATTAC H/industry/11Medical\% 20Services_CN.pdf,
[7] C. Lu, Z. W. Qi, S. Yu, and D. P. Su, "The medical center of mental patients discharged from the status of the investigation plan," Public Health, vol. 26, no. 2, pp. 103-113, 1999.

[8] C. Ling, Y. T. Dai, and M. F. Lou, "A coma patient's discharge planning services," Nursing, vol. 46, no. 5, pp. 93-100, 1999.

[9] The American Hospital Association, "Introduction to discharge planning for hospital," IL: American Hospital Association, 1983.

[10] M. D. J. Buss, "How to rank the computer projects," Harvard Business Review, January-February, 1989.

[11] M. J. Earl and B. Khan, "How the new is business process Redesign?" European Management Journal, vol. 12, no. 1, pp. 20-30, March 1994.

[12] M. J. Earl, Management Strategies, for Information Technology, Prentice Hall, 1989.

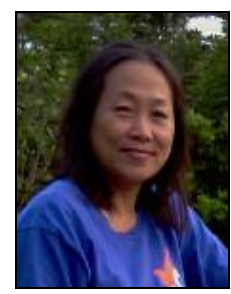

Liarn Rurng Wen was born in Pingtung, Taiwan. She received her master degree of science from Moorhead State University, USA in 1989. She is a senior instructor in Meiho University, Ping Tung, Taiwan. Her research areas are in health informatics and nursing informatics.

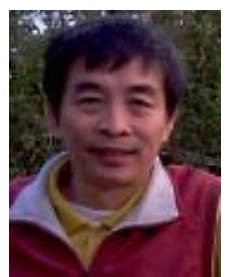

Ching Miin Duh was born in Taipei, Taiwan. $\mathrm{He}$ obtained his Ph.D. degree in management information systems from Royal Holloway University of London in 2001. He is an associated professor in National Taipei University of Nursing and Health Sciences, Taipei, Taiwan. His current research fields include health promotion management and healthcare knowledge management. 